\title{
Lower Third of the Esophagus
}

National Cancer Institute

\section{Source}

National Cancer Institute. Lower Third of the Esophagus. NCI Thesaurus. Code C12255.

The lower one third of the esophagus in which the muscle layer is composed of muscle cells predominantly of the smooth type. 